\title{
Germline mutations in a DNA repair pathway are associated with familial colorectal cancer
}

\author{
Pingping Xu, ${ }^{1}$ Danfeng Sun, ${ }^{1}$ Yaqi Gao, ${ }^{1}$ Yi Jiang, ${ }^{1}$ Ming Zhong, ${ }^{2}$ Gang Zhao, ${ }^{2}$ Jinxian Chen, ${ }^{2}$ \\ Zheng Wang, ${ }^{2}$ Qiang Liu, ${ }^{3}$ Jie Hong, ${ }^{1}$ Haoyan Chen, ${ }^{1}$ Ying-Xuan Chen, ${ }^{1}$ and Jing-Yuan Fang ${ }^{1}$ \\ ${ }^{1}$ State Key Laboratory for Oncogenes and Related Genes, Key Laboratory of Castroenterology \& Hepatology, Ministry \\ of Health, Division of Gastroenterology and Hepatology, Shanghai Institute of Digestive Disease, ${ }^{2}$ Department of Surgery, \\ and ${ }^{3}$ Department of Pathology, Renji Hospital, School of Medicine, Shanghai Jiao Tong University, Shanghai, China.
} \begin{abstract}
predisposition in familial colorectal cancer (CRC), we performed whole-exome sequencing in 24 familial CRCs. Mutations in genes that regulate DNA repair (RMI1, PALB2, FANCI) were identified that were related to the Fanconi anemia DNA repair pathway. In one pedigree, we found a nonsense mutation in CHEK2. CHEK2 played an essential role in cell cycle and DNA damage repair. Somatic mutation analysis in CHEK2 variant carriers showed mutations in TP53, $A P C$, and FBXW7. Loss of heterozygosity was found in carcinoma of CHEK2 variant carrier, and IHC showed loss of Chk2 expression in cancer tissue. We identified a second variant in CHEK2 in 126 sporadic CRCs. A KO cellular model for CHEK2 (CHEK2 ${ }^{\mathrm{KO}}$ ) was generated by CRISPR/Cas9. Functional experiments demonstrated that $\mathrm{CHEK} 2^{\mathrm{K} 0}$ cells showed defective cell cycle arrest and apoptosis, as well as reduced p53 phosphorylation, upon DNA damage. We associated germline mutations in genes that regulate the DNA repair pathway with the development of CRC. We identified CHEK2 as a regulator of DNA damage response and perhaps as a gene involved in CRC germline predisposition. These findings link CRC predisposition to the DNA repair pathway, supporting the connection between genome integrity and cancer risk.
\end{abstract}

Aiming to identify rare high-penetrance mutations in new genes for the underlying

Authorship note: PX and DS contributed equally to this work.

Conflict of interest: The authors have declared that no conflicts of interest exist.

Copyright: (c) 2021, Xu et al. This is an open access article published under the terms of the Creative Commons Attribution 4.0 International License.

Submitted: February 22, 2021

Accepted: July 30, 2021

Published: September 22, 2021

Reference information: /CI Insight. 2021;6(18):e148931. https://doi.org/10.1172/jici. insight.148931.

\section{Introduction}

Colorectal cancer $(\mathrm{CRC})$ is a common disease with a high mortality rate in the world. Germline predisposition and environmental factors affect CRC susceptibility. Importantly, the inherited germline contribution is known to influence about $12 \%-35 \%$ of all cases $(1,2)$. However, only $5 \%-7 \%$ of CRC cases are caused by germline mutations in genes that are responsible for Mendelian cancer syndromes. Lynch syndrome and familial adenomatous polyposis (FAP) are the most frequent forms of Mendelian CRC syndromes. Classic hereditary CRC syndromes are mainly due to germline mutations in $A P C, M U T Y H$, and the mismatch repair genes ( $M S H 2, M S H 6, P M S 2, M L H 1$; refs. 3, 4).

In addition to hereditary forms, around $30 \%$ of CRC cases also present familial aggregation — but with an unknown inherited cause. The hypothesis of rare high-penetrance mutations in genes yet to be discovered is a very likely explanation for the underlying predisposition in a portion of these familial CRC cases. Therefore, past efforts have been made in these familial CRC cases, and next-generation sequencing technologies added a new unbiased approach to facilitate the identification of new genes responsible for predisposition to CRC. New candidate genes related to CRC have been found, such as POLD1, POLE (encoding DNA polymerases), NTHL1 (encoding a base-excision repair protein), MSH3, GREM1, RNF43, RSP20, MLH3, FAF1, and MCM8 (5-14). However, a large part of the heritability of colorectal adenomatous polyposis and CRC remains unexplained and is widely postulated to be enshrined in unidentified, rare variants.

With the aim of identifying new hereditary CRC genes, we performed whole-exome sequencing (WES) in patients with familial CRC. Our final goal is to facilitate genetic counsel and to be able to correctly address prevention strategies in these families. 


\section{Results}

Clinical characteristics and germline sequencing results. We examined 24 individuals from 21 families with CRC or advanced colorectal adenoma (CRA) by WES (Supplemental Tables 1 and 2; supplemental material available online with this article; https://doi.org/10.1172/jci.insight.148931DS1). All individuals had strong disease aggregation compatible with an autosomal-dominant pattern of inheritance. To exclude known Mendelian cancer syndromes, all individuals were screened for mutations in $A P C, M U T Y H$, and mismatch repair genes (MSH2, MSH6, PMS2, MLH1) before WES analysis. All individuals were tested without mutations in known hereditary CRC, including FAP or Lynch syndrome.

WES was performed in all individuals with a mean coverage of $86 \times$ (Supplemental Table 3). Germline WES data analysis was selected for only vary rare variants $(<0.1 \%)$ producing a loss of function (LOF) or variants located on genes with a function compatible with cancer development $(7,8,10)$.

After filtering the variants, we detected LOF mutations in 6 different genes (RMI1, PALB2, FANCI, AMER1, CTNNB1, SGK2; Figure 1 and Table 1). Among this, we found 3 mutations related to the Fanconi anemia (FA) DNA repair pathway (RMI1, PALB2, FANCI; Table 1). We also found 3 mutations in POLE in 2 individuals. All variants were verified by Sanger sequencing (Supplemental Figure 1). Additional segregation for these variants was performed when available. Unfortunately, most of family members were not accessible (Figure 1).

In addition, we found 1 potentially pathogenic CHEK2 variant in the pedigree $\mathrm{K}$ (Figure $2 \mathrm{~A}$ ) that contained 2 individuals (II-1 and III-1) that had been sequenced. In this family, the proband (individual III-1) was first diagnosed with CRA at 50 years old and had recurrently multiple CRAs 2 and 7 years later, with the biggest adenoma being $1.5 \times 1.5 \mathrm{~cm}$ and tubular. The proband's father (individual II-1) was diagnosed with sigmoid colon adenocarcinoma at 73 years old due to ileus and underwent a colectomy. He was followed by colonoscopy every 6 or 12 months. At least 33 polyps were detected in his colon, with the biggest polyp being $2.5 \times 2.3 \mathrm{~cm}$, and they were predominantly advanced adenomas. The proband's brother (individual III-2) was first diagnosed with multiple CRAs at 49 years old. The proband's grandfather was diagnosed with gastric cancer at 70 years old and died several months after surgery. All the known members of this pedigree were not diagnosed with breast cancer.

We searched the sequences for genes that harbored damaging variants. After performing variant filtration and quality control, we filtered annotated variants and identified 3 heterozygous variants in 3 genes (Supplemental Table 4; Supplemental Table 5, A and B; Figure 2B; and Supplemental Table 6 for analysis flow chart). Two of these variants were missense mutations in $C H D 8$ and $M O B 1 B$, but the mutations were not found in the key domain of the protein. The third variant contained the highest Combined Annotation-Dependent Depletion (CADD) score (CADD score of 35) and was the only LOF variant. This CHEK2 (GenBank: NM_001005735) variant (hg19chr22: g.29130631 C>T) (ClinVar accession SCV001754535; https://www. ncbi.nlm.nih.gov/clinvar/) is predicted to cause a premature stop code in the SQ/TQ motif at position 27 (p.Gln27*, also named as p.Q27*; Figure 2C). Sanger sequencing further confirmed the variant in individuals II-1 and III-1 (Figure 2D), as well as another affected family member (III-2; Figure 2D). Segregation analysis revealed the presence of the same germline CHEK2 variant in affected family members (II-1, III-1, III-2) where 1 individual exhibited CRC and multiple CRAs (individual II-1), and 2 individuals exhibited multiple CRAs (individual III-1 and III-2; Figure 2A). The 1000 Genomes Project, Exome Aggregation Consortium (ExAC), and the Genome Aggregation Database (gnomAD) do not contain this variant, confirming the identified mutation as a rare event (ftp-trace.ncbi.nih.gov/1000genomes; https://gnomad.broadinstitute.org/). Hence, we consider this mutation (CHEK2 p.Q27*) as a good candidate for CRA/CRC.

Tumor analysis. Furthermore, we examined 7 tumors ( 6 adenomas and 1 carcinoma) from 3 carriers of the CHEK2_p. Q27* variant using WES analysis (Supplemental Tables 7 and 8) to explore somatic mutations in tumor tissues. All tumors were screened for $K R A S$ and $B R A F$ driver mutations, and a total of 83 cancer-related genes were screened, including APC, CTNNB1, PIK3CA, and FBXW7 (Supplemental Table 9 and refs. 15, 16). All tumors were microsatellite stable (MSS; Table 2 and Supplemental Table 8). As shown in Table 2, somatic mutations in tumor tissues were mainly found in $A P C, T P 53$, and FBXW7. For $A P C$, all mutations were LOF variants (nonsense and frameshift). There was also a missense mutation in TP53 (NM_001126112:c.742C>T, p.R248W) in the carcinoma tissues, suggesting that TP53 may be involved in the colorectal tumorigenesis in this case. The TP53 mutation was located in the DNA-binding domain, which is an important structural domain. However, mutations were not found in other pathogenic genes, including CTNNB1, KRAS, and BRAF.

Loss of heterozygosity (LOH) involving the germline WT allele was found in the cancer tissue of II-1 (Supplemental Table 8). However, IHC staining of Chk2 in the adenoma tissues of 3 CHEK2 variant carriers including 
A

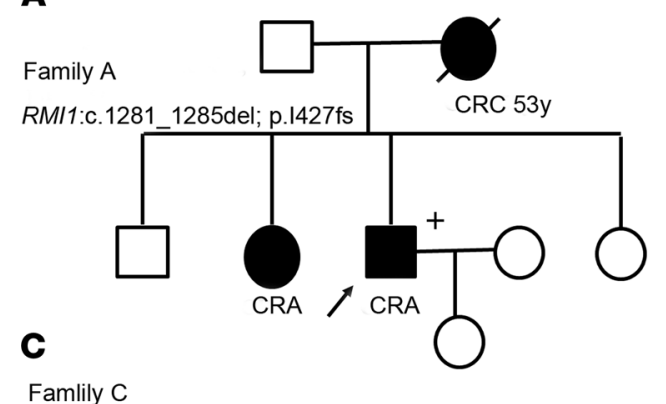

Famlily C

FANCl: c. 2960 C>T; p.T987M

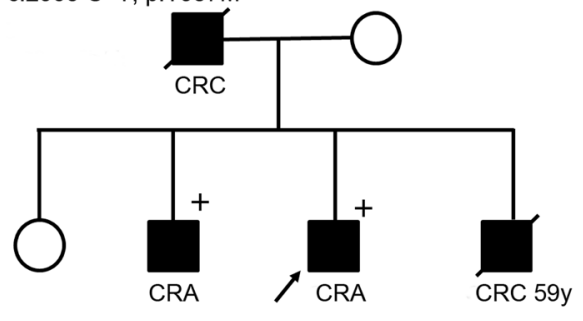

E

Famlily E

BRF1:c.1954 G>A; p.G652R

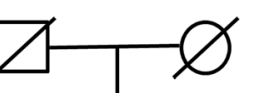

\section{B}

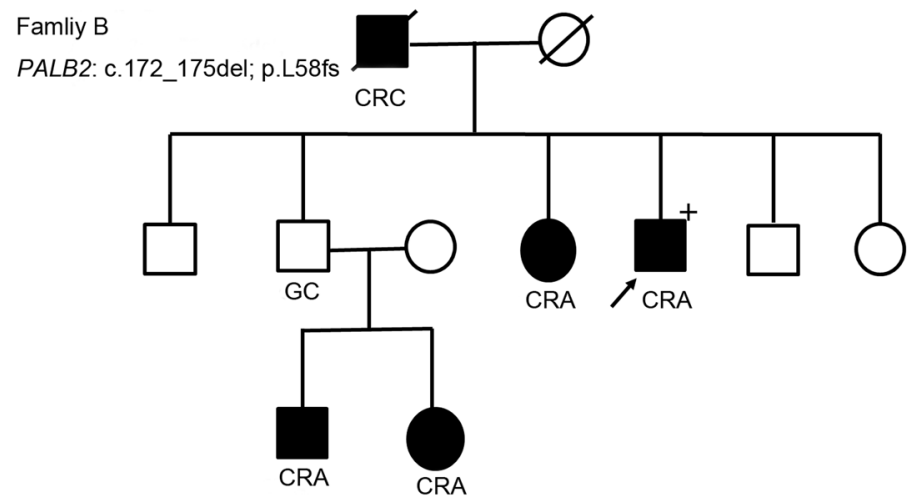

D

Famliy D

POLE:c.1187A>G; p.E396G

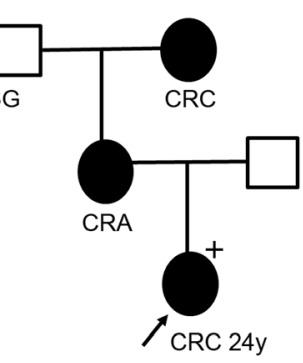

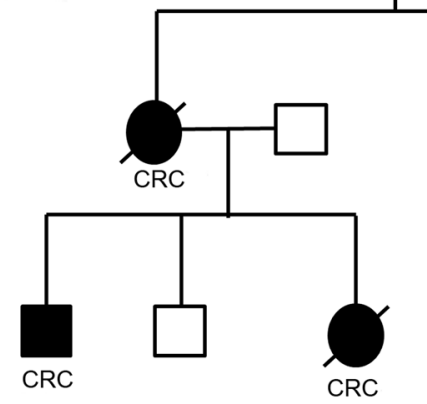

G

Famliy $G$

CTNNB1:C.1444C>G; p.Q482E

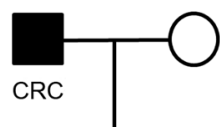

F

Famliy $\mathrm{F}$

POLE:c.2929G>A;p.G977R;

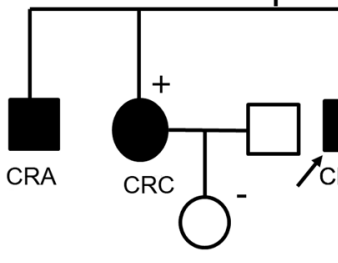

H

Famliy $\mathrm{H}$

AMER1:c.3145C>T; p.R1049
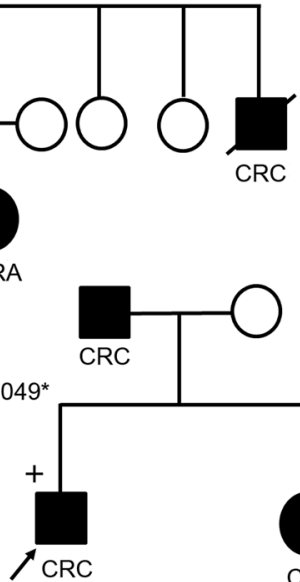

CRA
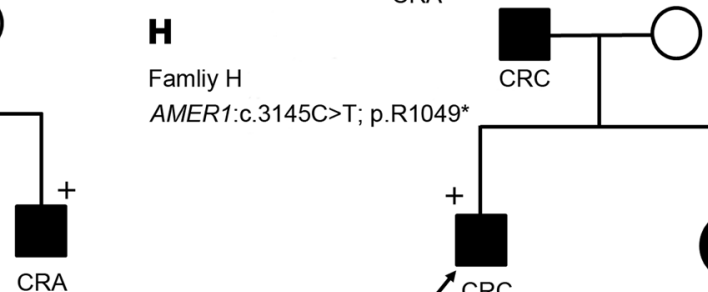

$\nearrow \mathrm{CRC}$
c.1346C >T; p.T449M

(a)

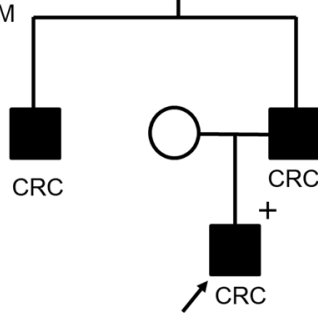

I

Famliy I SGK2:c.560G $>A$ p.R187Q

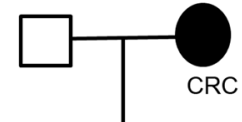

Figure 1. Pedigrees of study participants with strong family histories of CRC and the identifiable mutations. (A-I) Squares indicate male family members, and circles represent female members. A slash through a symbol indicates that the family member has died. Filled symbols indicate those affected by colorectal cancer or advanced colorectal adenomas. (+), mutation carrier; (-), nonmutation carrier. The proband is indicated by an arrow. The clinical details, including the features of the additional relatives and the number of tumors in those families, are shown in Supplemental Table 13.

individual II-1 revealed nuclear expression of the protein (Figure 3). It is noteworthy that in II-1's cancer tissue, Chk2 expressed in adjacent normal cells but not in cancer cells, indicating somatic inactivation of the WT allele in cancer cells. This is consistent with the CHEK2 LOH in cancer tissue detected by WES (Supplemental Table 8).

CRISPR/Cas 9 CHEK2 ${ }^{K O}$ modeling. As shown in pedigree $\mathrm{K}$, we found that the CHEK2 variant (p.Q27*) led to a premature stop at amino acid 27 and resulted in the loss of normal protein structure and function in Chk2. Using CRISPR/Cas9 technology, we generated CHEK2 knock-out $\left(C H E K 2^{\mathrm{KO}}\right)$ SW480 cells to reproduce the LOF mutant and further investigate the physiological role of Chk2 (Figure 4A). According to bioinformatic CRISPR prediction tools, a single-guide RNA (sgRNA) targeting the tenth exon was selected. The genotype 
Table 1. Description of the candidate variants

\begin{tabular}{|c|c|c|c|c|c|c|c|c|}
\hline Gene & Mutation & SNP ID & Protein effect & $\begin{array}{l}\text { CADD_ } \\
\text { PHRED }\end{array}$ & $\begin{array}{l}\text { ClinVar } \\
\text { accession no. }\end{array}$ & gnomAD_exome & Dormain/region & Function \\
\hline RMI1 & $\begin{array}{l}\text { c.1281_1285del } \\
\text { (p.1427fs) }\end{array}$ & rs764792608 & $\begin{array}{l}\text { Frameshift } \\
\text { deletion }\end{array}$ & & SCV001754808 & 4.18E-06 & & $\begin{array}{l}\text { Fanconi anemia } \\
\text { pathway, double- } \\
\text { strand break repair } \\
\text { via homologous } \\
\text { recombination }\end{array}$ \\
\hline PALB2 & $\begin{array}{l}\text { c.172_175del } \\
\text { (p.L58fs) }\end{array}$ & rs180177143 & $\begin{array}{l}\text { Frameshift } \\
\text { deletion }\end{array}$ & & SCV001754809 & $3.66 \mathrm{E}-05$ & & $\begin{array}{l}\text { Fanconi anemia } \\
\text { pathway, double- } \\
\text { strand break repair } \\
\text { via homologous } \\
\text { recombination }\end{array}$ \\
\hline FANCI & $\begin{array}{l}\text { c.2960C>T } \\
\text { (p.T987M) }\end{array}$ & rs138432305 & Missense & 26.4 & SCV001754757 & $2.84 \mathrm{E}-05$ & $\begin{array}{l}\mathrm{FANCI} \text { solenoid } 3 \\
\text { domain }\end{array}$ & $\begin{array}{l}\text { Fanconi anemia } \\
\text { pathway, interstrand } \\
\text { cross-link repair }\end{array}$ \\
\hline CHEK2 & $\begin{array}{l}\text { c.79C>T } \\
\left(\text { p.Q2 } 27^{*}\right)\end{array}$ & & Stopgain & 35 & SCV001754535 & & & $\begin{array}{l}\text { DNA damage } \\
\text { checkpoint }\end{array}$ \\
\hline SGK2 & $\begin{array}{l}\text { c.560G>A } \\
\text { (p.R187Q) }\end{array}$ & rs35187177 & Missense & 34 & SCV001754807 & $5.83 E-05$ & $\begin{array}{l}\text { Protein kinase } \\
\text { domain }\end{array}$ & $\begin{array}{l}\text { PI3K-Akt signaling } \\
\text { pathway }\end{array}$ \\
\hline CTNNB1 & $\begin{array}{l}\text { c.1444C>C } \\
\text { (p.Q482E) }\end{array}$ & & Missense & 23.8 & SCV001754805 & & $\begin{array}{l}\text { Armadillo-like } \\
\text { helical }\end{array}$ & $\begin{array}{l}\text { Wnt signaling } \\
\text { pathway }\end{array}$ \\
\hline AMER1 & $\begin{array}{l}\text { c. } 3145 C>T \\
\left(p . R 1049^{*}\right)\end{array}$ & & Stopgain & 36 & SCV001754806 & 5.96E-06 & & $\begin{array}{l}\beta \text {-Catenin destruction } \\
\text { complex assembly }\end{array}$ \\
\hline POLE & $\begin{array}{l}\text { c.1187A>G } \\
\text { (p.E396G) }\end{array}$ & & Missense & 27.8 & SCV001754759 & & $\begin{array}{l}\text { DNA-directed DNA } \\
\text { polymerase, family } \\
\text { B, exonuclease } \\
\text { domain }\end{array}$ & $\begin{array}{l}\text { Base-excision repair, } \\
\text { gap-filling,DNA } \\
\text { replication }\end{array}$ \\
\hline
\end{tabular}

of $C H E K 2^{\mathrm{KO}}$ clones was determined by Sanger sequencing (Figure 4B). The complete loss of Chk2 production in $C H E K 2^{\mathrm{KO}}$ cells was confirmed by Western blot analysis (Figure $4 \mathrm{C}$ ). We found that there was no influence on cell proliferation after CHEK2 KO (Figure 4D). This indicated that this CRISPR cell model can be used to explore the functional characterization of $C H E K 2$.

Functional characterization of germline variants. $C H E K 2^{\mathrm{KO}}$ cells show a defect in $\mathrm{G} 2$ cell cycle arrest after DNA damage. Given that Chk2 is activated in response to DNA damage and may regulate cell cycle arrest, we treated $C H E K 2^{\mathrm{WT}}$ and $C H E K 2^{\mathrm{KO}} \mathrm{SW} 480$ cells with nocodazole, a microtubule-disrupting agent that traps cells in mitosis. When cells were treated with nocodazole at different concentrations, the expression of phospho- $\gamma \mathrm{H} 2 \mathrm{AX}$ was elevated when compared with the untreated cells, and DNA damage was induced (Supplemental Figure 2A). When cells were treated with nocodazole for 6 hours, the cell cycle was arrested and about $50 \%$ of $C H E K 2^{\mathrm{WT}}$ and $C H E K 2^{\mathrm{KO}}$ cells were arrested in $\mathrm{G} 2$ phase (Figure 5 , A and B). After 12 hours, more $C H E K 2^{\mathrm{KO}}$ cells entered $\mathrm{G} 1$ and $\mathrm{S}$ phases relative to $C H E K 2^{\mathrm{WT}}$ cells. These results indicated that there was a defect in $\mathrm{G} 2$ cell cycle arrest in $C H E K 2^{\mathrm{KO}}$ cells after DNA damage.

CHEK2 ${ }^{K O}$ cells were resistant to DNA damage-induced apoptosis and influence p53 phosphorylation during DNA damage. If damaged DNA cannot be repaired, cells can initiate apoptosis, which may also be regulated through the Chk2 kinase. To investigate the role of Chk2 in apoptosis, Adriamycin (which can induce double-stranded DNA breaks) was used to treat $C H E K 2^{\mathrm{WT}}$ and $C H E K 2^{\mathrm{KO}}$ cells. When $C H E K 2^{\mathrm{WT}}$ cells were treated 
A

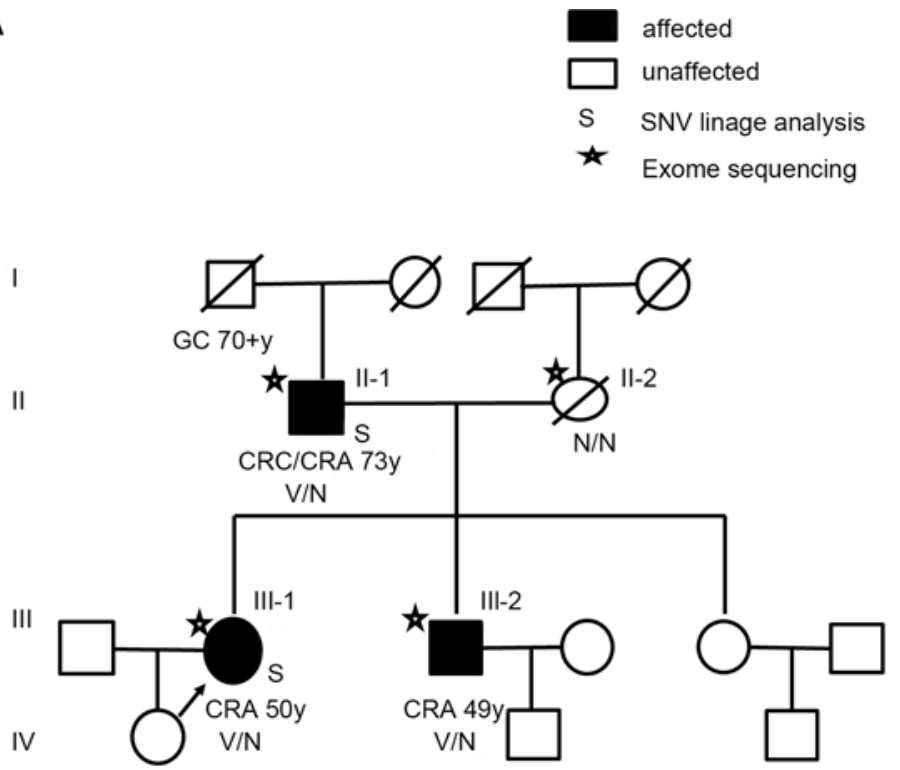

C

CHEK2 WT

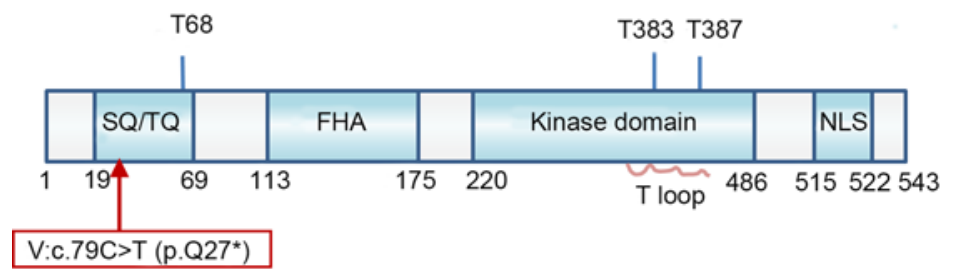

B

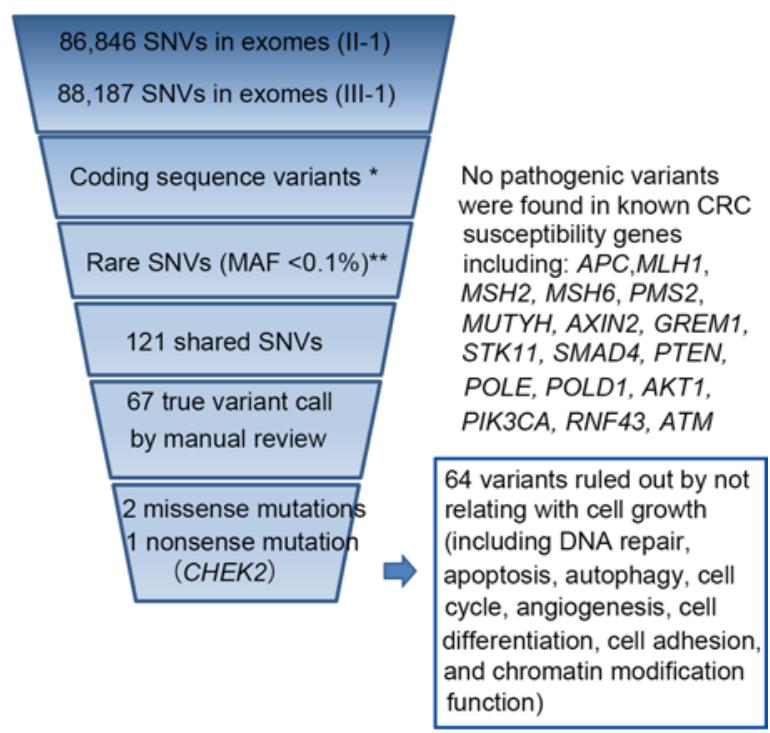

D

II-1

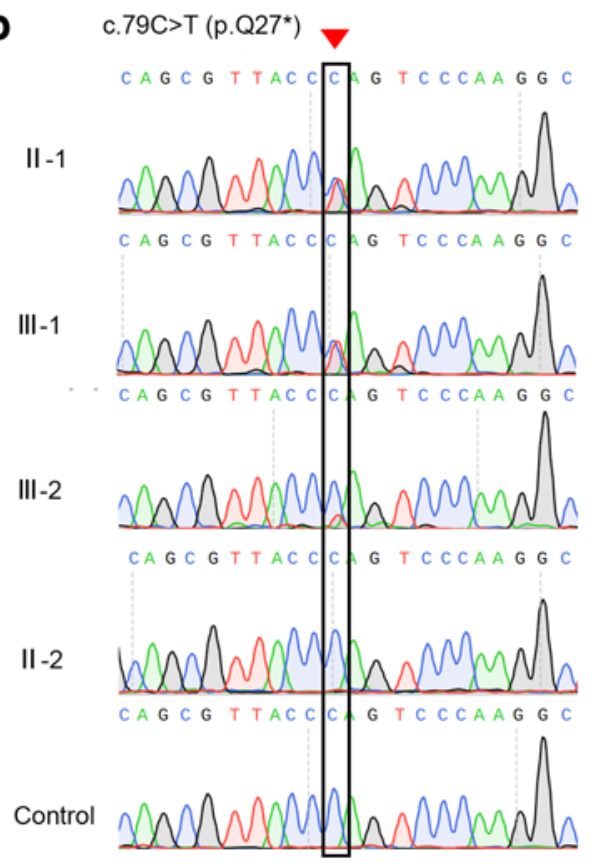

Figure 2. Gene discovery and characterization. (A) Pedigree K. Squares indicate male family members, and circles represent female members. A slash through a symbol indicates that the family member has died. Filled symbols indicate a clinically affected family member. The proband is indicated by an arrow. WES analysis was performed on 2 individuals, which are marked by the letter S. The CHEK2_p.Q27* mutant germline allele that was detected by Sanger sequencing is shown below each individual. " $\mathrm{V} / \mathrm{N}$ " indicates a heterozygous variant carrier, and "N/N" indicates a noncarrier. Age of tumor diagnosis is shown beneath each symbol. (B) The filter-based computational algorithm that is used to narrow candidate variants for pedigree K. Single-nucleotide variant, SNV; minor allele frequency, MAF. (C) The functional domains of Chk2 and the predicted truncated Chk2 protein that would result from the variant. SQ/TQ indicates the SQ/TQ motif, which is the consensus site for phosphoinositide-kinase-related kinases (PIKKS). FHA indicates the forkhead-associated domain. NLS indicates the nuclear localization signal. (D) Sanger sequencing-based validation of the germline CHEK2_p.Q27* mutation in individuals of this pedigree. The red arrowhead and black box indicate the location of the heterozygous substitution of $C$ to $T$ in the mutated locus of CHEK2.

with Adriamycin $(6 \mu \mathrm{M})$, around $69 \%$ and $57 \%$ of cells were viable after 24 and 48 hours, respectively (Figure 5, C and D, and Supplemental Figure 2B). With the same concentration of Adriamycin, around $80 \%$ and $71 \%$ of $C H E K 2^{\mathrm{KO}}$ cells were viable after 24 and 48 hours, respectively (Figure 5, C and D, and Supplemental Figure 2B). There was a significantly lower percentage of apoptotic cells in Adriamycin-treated $C H E K 2^{\mathrm{KO}}$ cell samples compared with Adriamycin-treated $C H E K 2^{\mathrm{WT}}$ cell samples (Figure 5, C and D). The crystal violet 
Table 2. Summary of somatic changes in CHEK2 p.Q27* mutation carriers' colorectal tumors

\begin{tabular}{lcc}
\hline Subject & Somatic mutation & Protein alteration \\
II-1 & TP53 c. C742T & p53 p. R248W \\
& APC c.C646T & APC p.R216X \\
& APC c.C4285T & APC p.Q1429X \\
& APC c. C4189T & APC p.E1397X \\
III-1 & APC c.3005delC & APC p.A1002fs \\
& APC c.4270delC & APC p.P1424fs \\
& FBXW7 c.C1513T & FBXW7 p.R505C \\
III-2 & None found & None found
\end{tabular}

assay also confirmed that Adriamycin-treated $C H E K 2^{\mathrm{KO}}$ cells had higher cell viability than Adriamycin-treated $C H E K 2^{\text {WT }}$ cells. As the concentration of Adriamycin increased, the difference in cell survival between $C H E$ $K 2^{\mathrm{WT}}$ and $C H E K 2^{\mathrm{KO}}$ cells became apparent (Figure 5, E and F). $C H E K 2^{\mathrm{KO}}$ cells were also resistant to apoptosis induced by other agents that induce DNA damage, such as nocodazole (Figure 5E and Supplemental Figure 2, $\mathrm{C}$ and D). This result indicates that $C H E K 2^{\mathrm{KO}}$ cells were resistant to DNA damage-induced apoptosis.

It has been reported that Chk2 regulates cell cycle arrest and apoptosis during the DNA damage response through p53. Chk2 can directly phosphorylate p53 on serine 20 to disrupt its association with Mdm2, thus promoting its stability (17-19). To explore the function of Chk2 in p53 regulation, the protein level of p53 after DNA damage was assessed. As shown in Figure 5G, $C H E K 2^{\mathrm{WT}}$ cells expressed high protein levels of phosphorylated p53 on serine 20 when treated with Adriamycin. In contrast, $C H E K 2^{\mathrm{KO}}$ cells had reduced levels of pan-p53 and phosphorylated p53 compared with $C H E K 2^{\mathrm{WT}}$ cells (Figure 5G). This result suggests that Chk2 may influence 553 phosphorylation during DNA damage.

Screening of the candidate gene variants in an independent cohort. In order to investigate the frequency of CHEK2 in sporadic CRC/CRA and normal populations, we genotyped another cohort to screen for the mutation encoding the CHEK2_p.Q27* variant. The cohort included 352 individuals of Chinese ancestry with colorectal tumors (including 230 individuals with CRC and 122 individuals with CRA; Supplemental Table 10). For comparison, we genotyped 100 control individuals, who were of Chinese ancestry but had not been diagnosed with polyposis or CRC (Supplemental Table 10). We found no additional cases or no controls that were heterozygotes for the mutation encoding the CHEK2_p.Q27* variant, further confirming CHEK2_p.Q27* was a rare event.

We screened the entire CHEK2 coding sequence in the germline DNA from 126 sporadic CRC patients by Sanger sequencing. An additional rare missense variant (NM_001005735:c.766 C>G, p. P256A) was detected in these sporadic cases (Supplemental Figure 1). This identified missense variant was not found in a large population dataset (gnomAD; https://gnomad.broadinstitute.org/)) and was predicted to be potentially pathogenic in silico tools (e.g., SIFT prediction: deleterious) (http://provean.jcvi.org/index.php). However, segregation for the detected genetic variant was not available in the family.

\section{Discussion}

In this study, we identified potential CRC predisposition variants in genes (RMI1, PALB2, FANCI, and $C H E K 2$ ) that regulate the DNA damage response, including the FA DNA repair pathway and the cell cycle.

It is widely held that genomic instability is a prerequisite for cancer formation. Colorectal epithelial cells are recurrently exposed to endogenous and exogenous mutagens and having high turnover rates (20). Therefore, DNA damage repair (DDR) mechanisms are fundamental to maintain the genomic integrity of the cell. The malfunctioning of DDR is strongly associated with carcinogenesis agents. Checkpoint mechanisms serve a major regulatory function in governing the DDR and ensure the coordination of DNA repair proteins, which detect and repair DNA damage to protect cells from genome instability.

FA is an inherited genomic instability disorder that contains bone marrow failure, growth abnormalities, and cancer predisposition. FA patients have chromosome fragility and hypersensitivity to drugs that induce DNA interstrand crosslinks (ICLs; ref. 21). The FA repair pathway is thought to coordinate a complex mechanism that contains elements of 3 classic DNA repair pathways in response to genotoxic insults. These 3 pathways include homologous recombination, nucleotide excision repair, and mutagenic translation synthesis (22). When the FA pathway is impaired, cells are hypersensitive to DNA damage and are unable to 
II-1 CRC
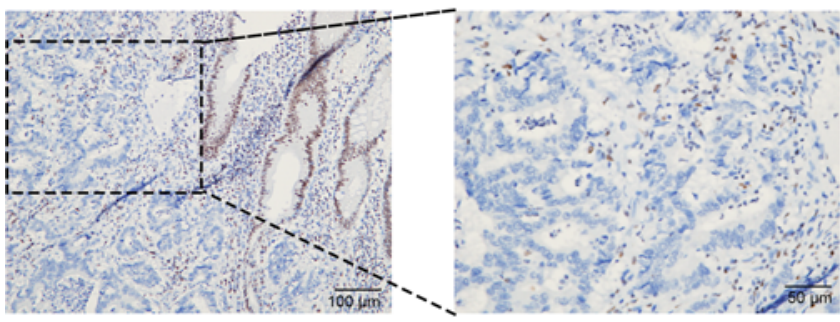

II-1 CRA

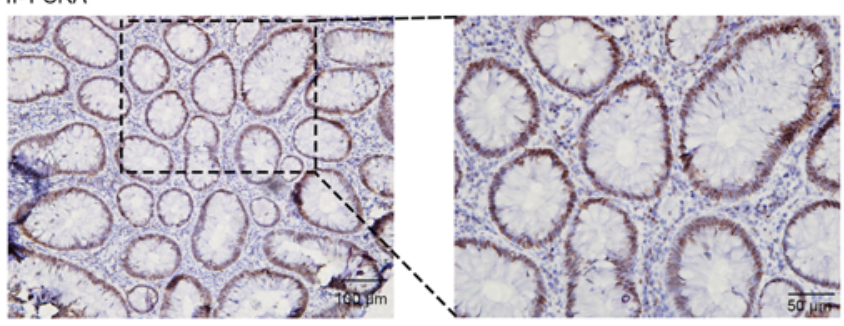

III-1 CRA

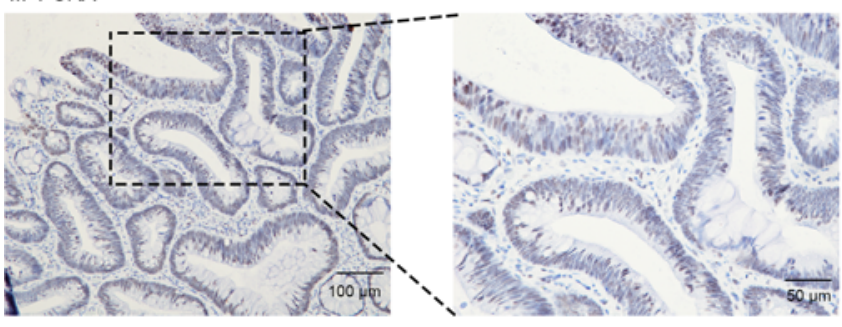

III-2 CRA

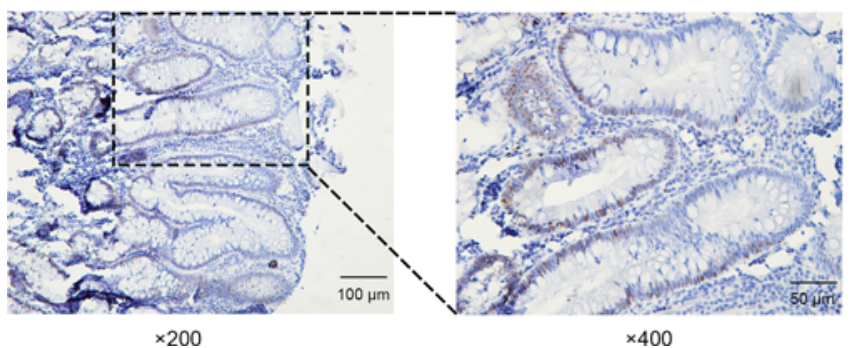

CHEK2 WT CRC

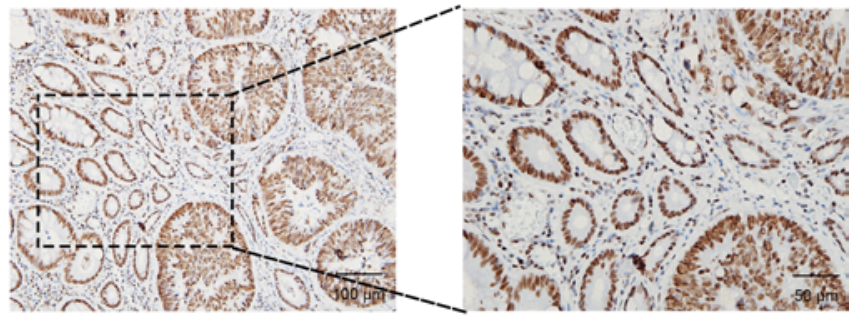

CHEK2 WT CRC normal adjacent

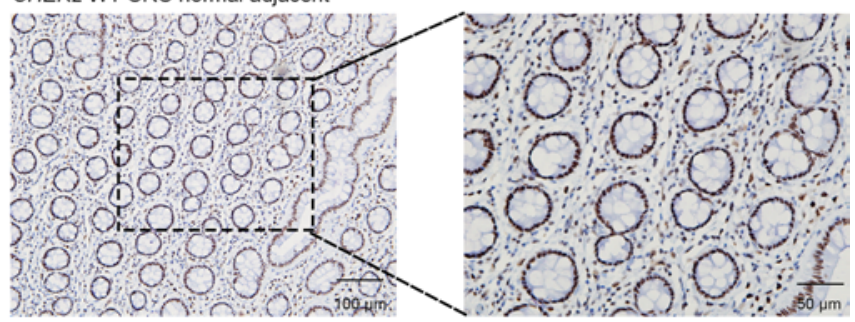

CHEK2 WT CRA

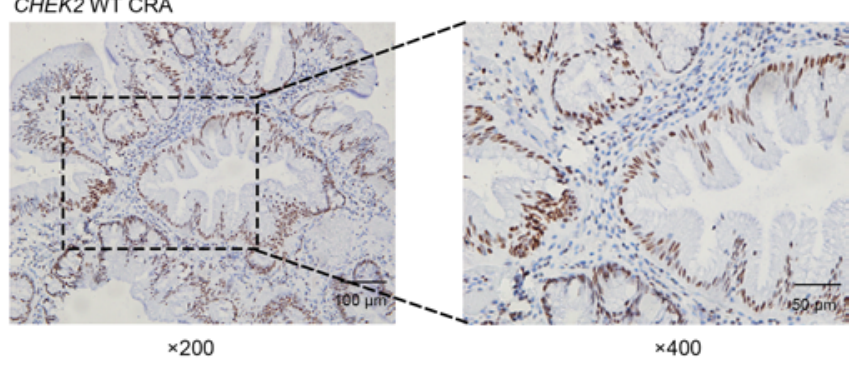

Figure 3. Chk2 IHC staining in the colorectal tumors developed by mutation carriers or WT controls. Representative photos of Chk2 IHC staining. Left, CHEK2 mutation carriers II-1, III-1, and III-2. Right, CHEK2 WT controls. Note the cancer cells with no nuclear staining, whereas the adjacent normal cells show strong staining in family member II-1. Scale bars: $50 \mu \mathrm{m}$ or $100 \mu \mathrm{m}$, as indicated.

successfully repair damaged DNA and cause genome instability. Mutations in the FA proteins lead to a high tumor incidence. Previous studies have indicated that malfunctioned FA genes and proteins have been found to be associated with a variety of cancers (23-27).

Previous studies have found FANCD2/FANCI-associated nuclease 1 gene (FAN1) mutations in the inherited susceptibility to CRC (28). In our study, by exome sequencing, we identified 3 potential CRC predisposition variants ( $R M I 1, P A L B 2, F A N C I)$ that were involved in the FA repair pathway.

RecQ-mediated genome instability protein 1 (RMI1) is an essential component of the RMI complex that plays an important role in the processing of homologous recombination. The RMI1 mutation (NM_024945: c.1281_1285del, p.I427fs) (ClinVar accession SCV001754808) is predicted to cause a premature stop and loss of protein function. It is likely to contribute to genomic instability. It has been reported that the RMI1 gene polymorphisms were associated with the risk of cancer $(29,30)$, but further studies are needed to investigate.

Partner and localizer of $B R C A 2$ ( $P A L B 2$, also termed FA complementation group N [FANCN]) plays a critical role in homologous recombination repair (HRR) through its ability to recruit BRCA2 and RAD51 to DNA breaks. Mutations in $P A L B 2$ have been reported in breast cancer, Fanconi anemia subtype FA-D1, and pancreatic cancer $(25,26,31-33)$. Previous studies also identify the association between $P A L B 2$ mutations and early-onset CRC (34). In our study, the PALB2 mutation (NM_024675: c.172_175del, p.L58fs) (ClinVar accession SCV001754809) is predicted to abolish protein function, thus causing the LOF in the HRR. It is likely to cause the impaired FA repair pathway and contribute to tumorigenesis. 
A

sgRNA3

AGCCAAGAGCATCTGGTAAAAATAGAGCTTGCAGGTAGCTT

TCGGTTCTCGTAGACCATTTTTATCTCGAACGTCCATCGAA

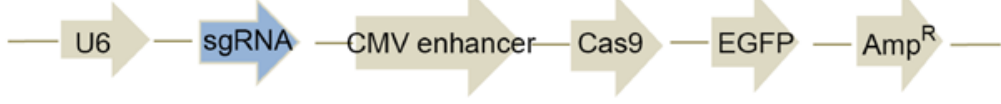

B

SW480

I GCTACCTGCAAGCTCTATTTTTAC

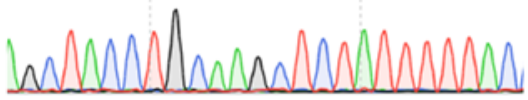

AGCTACCTOCAAGCTCTATTTTTAC

Mixed pool

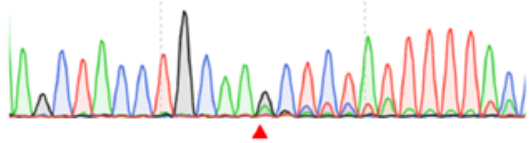

GCTACCTGCAAAGCTCTATTTTTA

$\mathrm{CHEK2}^{\mathrm{KO}}$

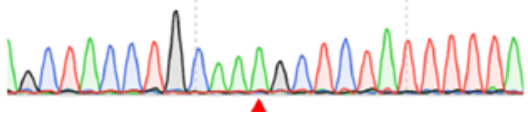

C

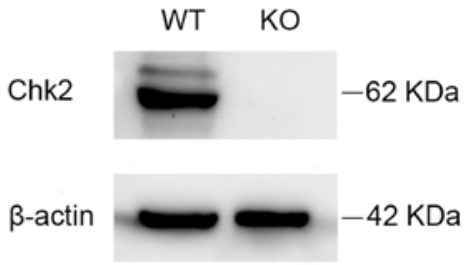

D

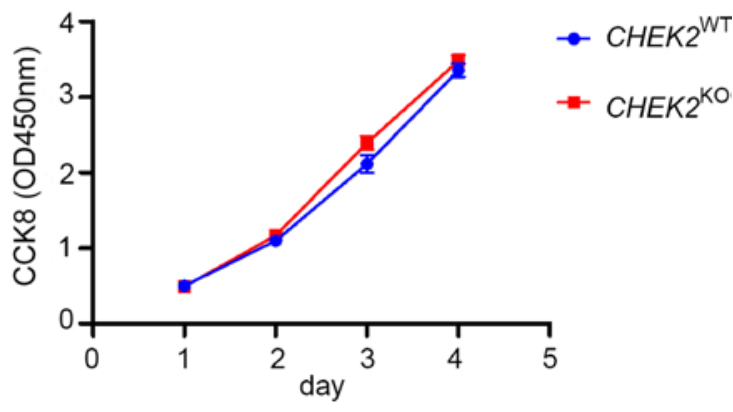

Figure 4. CRISPR/Cas9-mediated CHEK2 gene inactivation in CRC cell lines. (A) CRISPR-directed CHEK2 inactivation was designed targeting exon 10. (B) The aberrant sequence signal after the expected break site of the edited cell pool by Sanger sequencing (red arrowhead). (C) Chk2 protein level of the selected $C H E K 2^{\mathrm{K} 0}$ clones assessed by Western blot. (D) Proliferation of CHEK2 $2^{\mathrm{WT}}$ and $C H E K 2^{\mathrm{K} 0}$ cells detected by CCK 8 after cultured for the indicated time. Data are expressed as mean \pm SEM $(n=6)$, nonparametric Mann-Whitney $U$ test.

FA Complementation Group I ( $F A N C I$ ) plays an essential role in the repair of DNA double-strand breaks by homologous recombination. It takes part in the repair of DNA ICLs with FANCD2. It has been reported that FANCI mutations possibly involved in breast cancer and ovarian cancer susceptibility (27, 35). The FANCI mutation (NM_001113378: c.2960C>T, p.T987M) (ClinVar accession SCV001754757) is located inside the FANCI solenoid 3 domain. In silico pathogenicity tools predicted it as a possible pathogenic mutation. Because the unique nuclear protein complex that ubiquitinates FANCD2 and FANCI leads to formation of DNA repair structures, it is postulated that they may affect cancer risk in a specific manner.

FA repair pathway plays an important role in maintaining the genome stability; cell cycle regulations also are critical for DDR. In our study, we also identified a rare genetic variant with plausible pathogenicity in the CHEK2 gene in 3 individuals from 1 family with CRC. A functional characterization of mutation was performed in CRISPR/Cas9 cellular model to further confirm the pathogenicity and involvement in germline predisposition to CRC.

The serine/threonine protein kinase Chk2 encoded by CHEK2 is activated in response to DNA damage and subsequently regulates downstream effector proteins, including $\mathrm{p} 53, \mathrm{BRCA} 1$, and BRCA2, which are critical for DNA repair, cell cycle regulation, and cellular apoptosis $(36,37)$. Given the critical role of mitosis in cell survival, defects in cell cycle regulation may lead to abnormal cell division during DNA damage. Thus, germline mutation in CHEK2 may cause genomic instability and lead to cancer predisposition. Germline CHEK2 variants were first reported in families with Li-Fraumeni syndrome that lack TP53 mutations (38). Later, numerous studies reported that $C H E K 2$ is a multiorgan cancer susceptibility gene, such as breast (39), ovarian (40), prostate (41), and renal cancer (42). Recently, a multicenter case-control analysis using WES provided evidence for germline CHEK2 LOF variants as new moderate-penetrance variants in testicular germ cell tumors (43). Although it has been reported that CHEK2 I157T associates with an increased risk of CRC (44), no causal germline mutation of CHEK2 in CRC has been identified. In our study, a new germline CHEK2 LOF mutation was found in a CRC family, which is unavailable in the gnomAD database. The identified LOF mutation in CHEK2 (CHEK2_p.Q27*) is predicted to cause a premature stop codon in the $\mathrm{SQ} / \mathrm{TQ}$ motif, and in silico pathogenicity tools showed this mutation has the highest CADD score. We 
A

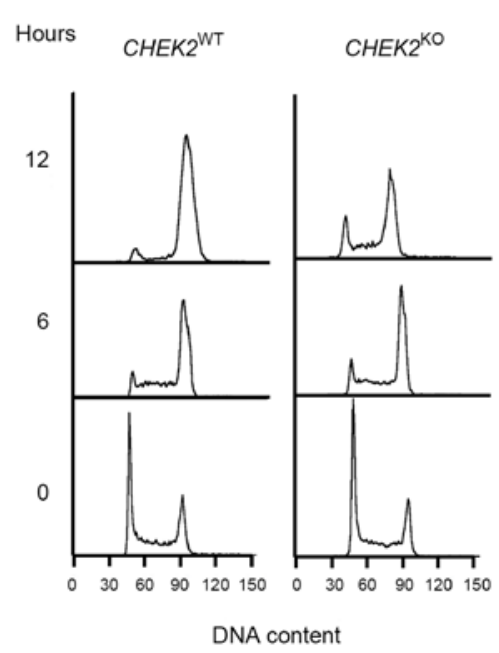

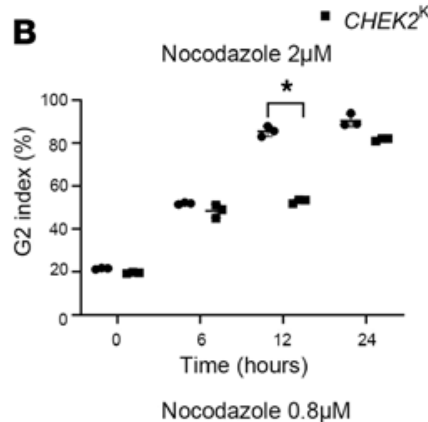

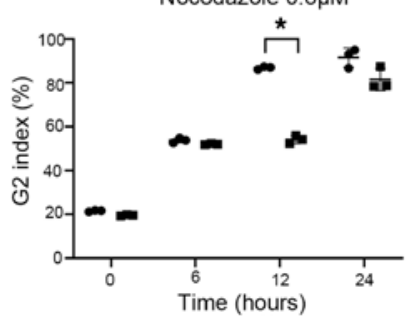

\section{E}

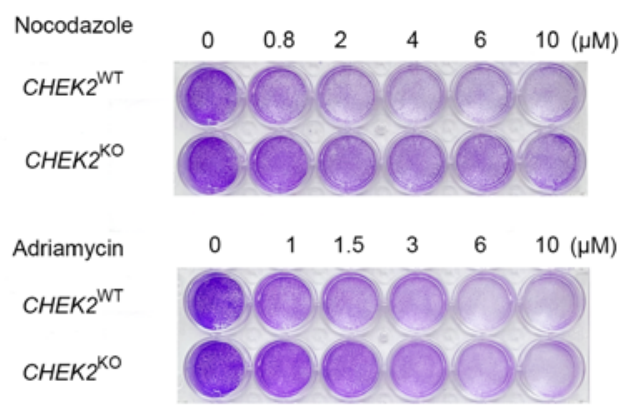

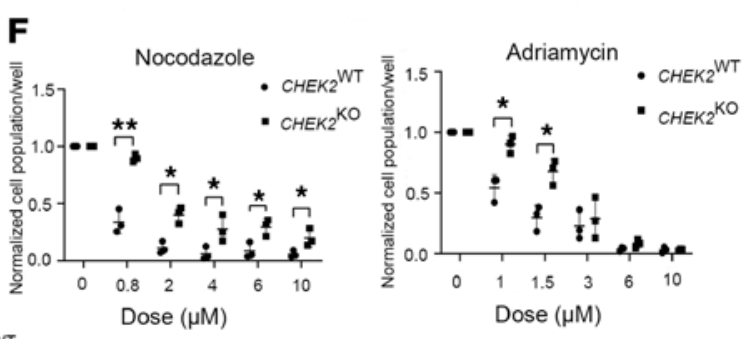

C
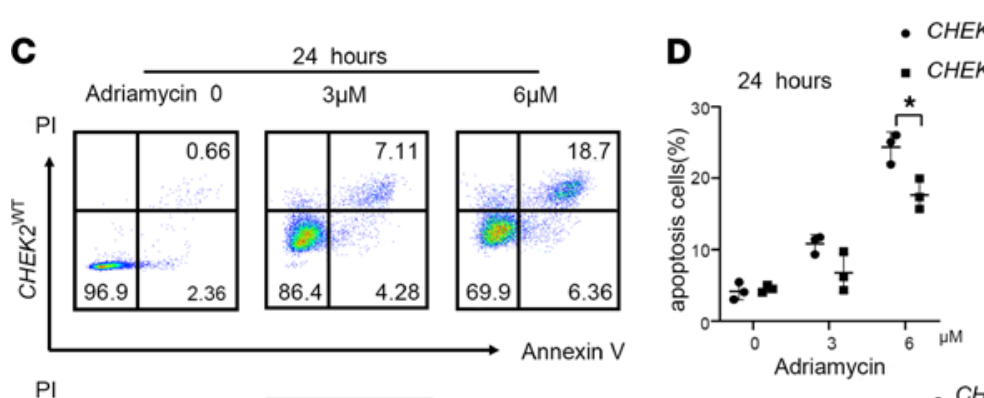

- $C H E K 2^{\mathrm{WT}}$

Dose $(\mu \mathrm{M})$

Dose $(\mu \mathrm{M})$
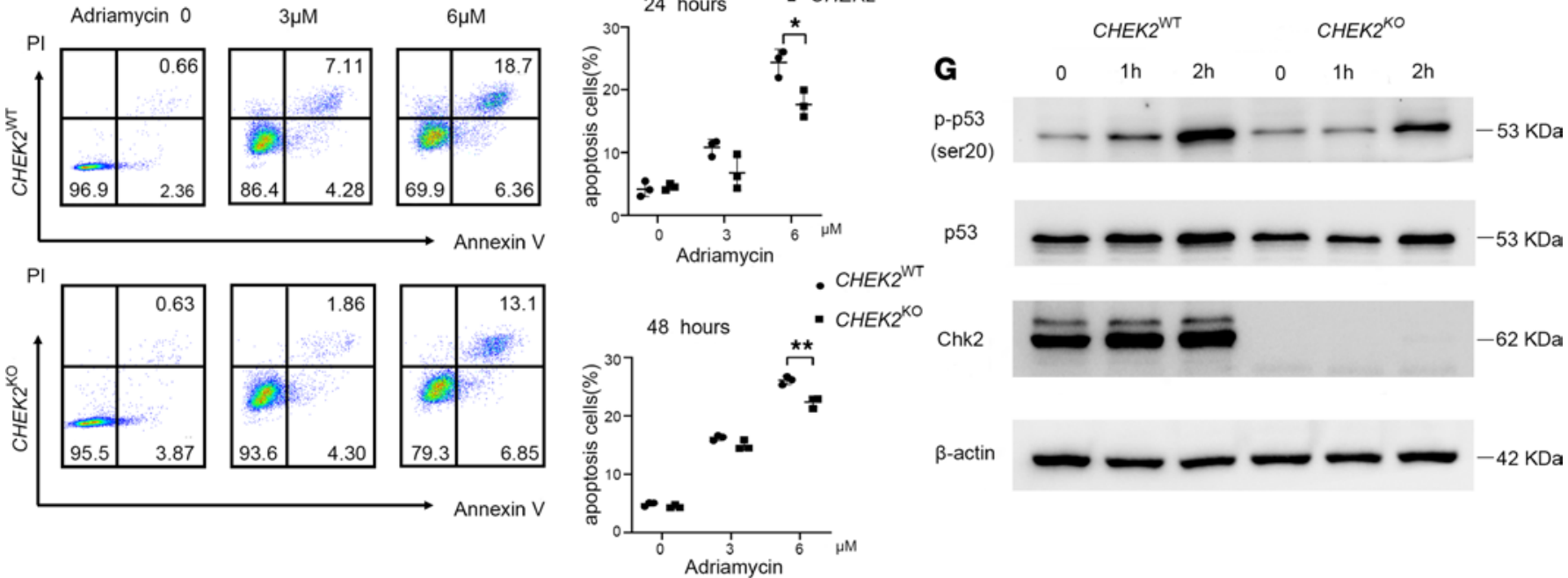

Chk2

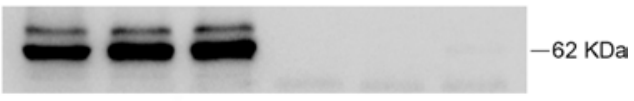

$\beta$-actin
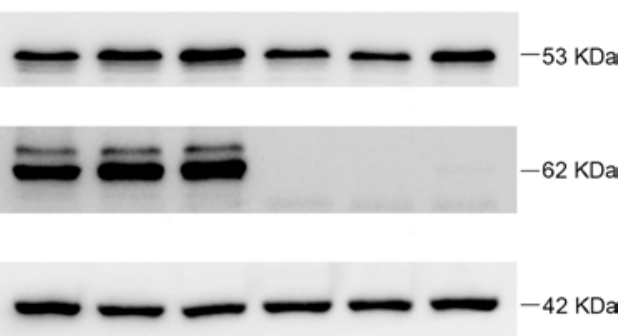

Figure 5. Failure of maintenance of nocodazole-induced G2 arrest and impaired DNA damage-induced apoptosis in CHEK2 ${ }^{\mathrm{KO}}$ cells. (A) Kinetics of cell cycle progression of $C H E K 2^{W T}$ and $C H E K 2^{\mathrm{KO}}$ cells after nocodazole treatment $(2 \mu \mathrm{M})$ for 0,6 , and 12 hours. (B) The percentage of cells that arrest at $\mathrm{C} 2$ after nocodazole treatment at different time points and concentrations. Data are expressed as mean $\pm \operatorname{SEM}(n=3) .{ }^{*} P<0.05$, nonparametric Mann-Whitney $U$ test. (C) Cellular apoptosis analysis by flow cytometry in $C H E K 2^{\mathrm{WT}}$ and $C H E K 2^{\mathrm{K} 0}$ cells treated with different concentrations of Adriamycin after 24 hours. (D) The percentage of apoptotic cells calculated by flow cytometry from C. Data are expressed as mean $\pm \operatorname{SEM}(n=3) .{ }^{*} P<0.05$, ${ }^{* *} P<0.01$, nonparametric Mann-Whitney test. (E) Cell viability detected by a crystal violet assay after cells were treated with different concentrations of nocodazole or Adriamycin. (F) Normalized cell population/well calculated by ImageJ software $(\mathrm{NIH})$ from $\mathbf{E}$. Data are expressed as mean $\pm \operatorname{SEM}(n=3) .{ }^{*} P<0.05$, nonparametric Mann-Whitney $U$ test.

(C) Protein levels of pan-p53, phosphorylated p53 (serine 20), and Chk2 in CHEK2 ${ }^{\mathrm{WT}}$ and CHEK2 ${ }^{\mathrm{KO}}$ cells after Adriamycin treatment for the indicated times.

also identified another rare genetic variant (NM_001005735:c. 766 C > G, p. P256A) in CHEK2 in 126 sporadic CRCs. These mutations in $C H E K 2$ may represent a genetic cause of intestinal neoplasia and cancer.

It is also known that Chk2 is activated during DNA damage and subsequently inhibits CDC25C phosphatase, preventing cells from entering mitosis, and stabilizes p53, resulting in a cell cycle arrest in G1. In our study, by performing CHEK2 gene editing in a cellular model, we were able to demonstrate its plausible effect on cell cycle arrest and maintain genomic integrity. Consistent with previous reports, $C H E K 2^{\mathrm{KO}}$ cells were unable to effectively maintain cell cycle arrest in the G2 phase with nocodazole treatment and were resistant to apoptosis after DNA damage. Lower levels of pan-p53 and phosphorylated p53 were detected in $C H E K 2^{\mathrm{KO}}$ cells compared with WT cells when double-strand DNA breaks 
were induced. These results are consistent with the view that Chk2 plays an important role in cell cycle regulation and genomic integrity maintenance.

Loss of cell cycle checkpoint capacity caused by CHEK2 mutant genotypes may lead to specific somatic mutations in affected colon tissues. In our study, we found $A P C$ somatic mutations in adenomas of $C H E K 2$ mutant carriers. $A P C$ somatic mutations have been shown to precede other germline gene mutation carriers in CRC or adenomas $(7,8,12)$. CHEK2 LOH was found in the cancer tissue of II-1, and IHC staining showed Chk2 expressed in adjacent normal cells but not in cancer cells, indicating second-hit somatic inactivation of the WT allele in cancer cells. In addition, in this pedigree, we also observed a preferential development of CRC with TP53 mutations in germline CHEK2 mutant carriers, where CHEK2 second-hit inactivation may precede TP53 mutation. In previous studies, somatic mutations in $K R A S, B R A F$, and PIK3CA have been found in $\mathrm{CRC}$ or adenomas from other germline gene mutation carriers. But in our study, somatic mutations were not found in these genes, suggesting that the tumors did not follow the pathway of colorectal tumorigenesis induced by somatic mutations in $K R A S, B R A F$, and $P I K 3 C A$. It has been reported that Chk2 can stabilize p53 during DNA damage. A possible mechanism is that the LOF Chk2 could not effectively stabilize p53.

Besides the genes related to the FA DNA repair pathway or the cell cycle, we also found another 2 genes (CTNNB1 and AMER1; CTNNB1:c.1444C>G;p.Q482E, AMER1:c.3145C>T; p.R1049*; ClinVar accessions SCV001754805 and SCV001754806) related to the Wnt signaling pathway. AMER1 is located on chromosome $\mathrm{X}$, and the mutation carrier was a male patient. Therefore, the mutation in $A M E R 1$ was a homozygous variant. It is well known that the Wnt signaling pathway is strongly associated with colorectal carcinogenesis. Although somatic mutations in CTNNB1 occur frequently in colon cancer, germline mutations have been less implicated in hereditary CRC. This finding may indicate that germline mutations in CTNNB1 or AMER1 may be the candidate genes for CRC. Additionally, we also found a missense mutation in $S G K 2$ that took part in the PI3K-Akt signaling pathway. We also identified rare variants in $P O L E$ and $B R F 1$, which have been reported as the pathogenic genes $(7,45)$. Among this, 1 patient carrying the POLE variant was an early-onset CRC. Another patient carried compound heterozygous mutations in POLE. This confirmed that $P O L E$ takes part in the colorectal tumorigenesis.

In summary, our results highlight some candidate genes for CRC germline predisposition, which involved in DNA repair and the cell cycle. Our findings implicate germline CHEK2 mutations in the inherited susceptibility to CRC, as well as the defective cell cycle arrest and apoptosis, as the plausible underlying mechanism. Our results further support the relationship between DNA repair and cancer predisposition.

\section{Methods}

Patients. We selected 24 individuals from 21 families. All of these individuals had strong CRC aggregation, but other known germline alterations of hereditary cancer syndromes (FAP and Lynch syndrome) tested negative. APC and MUTYH were tested by Sanger sequencing to identify FAP. Lynch syndrome was excluded by IHC to test the expression of MSH2, MSH6, PMS2, and MLH1. These individuals fulfilled the following criteria: they had 2 or more relatives with CRC or CRA, and 2 or more consecutive affected generations, and all individuals screened negative for FAP and Lynch syndrome. Family members were included for segregation analysis of genetic variants.

In order to investigate the frequency of candidate mutations in sporadic CRC/CRA and normal populations, 100 healthy controls who were of Chinese ancestry but had not been diagnosed with polyposis or CRC and 352 individuals with colorectal tumor (CRC/CRA) were recruited from Renji Hospital, School of Medicine, Shanghai Jiao Tong University, for further variant genotyping.

DNA extractions and WES. Peripheral-blood genomic DNA was extracted by QIAamp DNA Blood Kit (Qiagen). WES was performed in individuals, similar to previous reports with some modifications (46). In brief, whole-exome capture and library preparation were performed using the Twist Fast Hybridization target enrichment system. The captured library was sequenced on the Illumina HiSeq platform (Illumina Nova 6000) according to the manufacturer's protocol. Subsequently, reads were trimmed and mapped to the human hg19 genome reference assembly with Burrows-Wheeler Alignment (BWA) and sorted by Picard-tools. Single-nucleotide variants (SNVs) and indel variants were called with Genome Analysis Toolkit (GATK). The variants were further annotated by ANNOVAR with 1000 Genomes Project, gnomAD, ExAC, SIFT, PolyPhen2 (http://genetics.bwh.harvard.edu/pph2/dokuwiki/overview), MutationTaster (http://www.mutationtaster.org/), CLINVAR, CADD (http://cadd.gs.washington.edu/score), COSMIC (http://cancer.sanger. ac.uk/cancergenome/projects/cosmic/), Generic mutation, GO terms (http://www.geneontology.org/), KEGG pathway (http://www.genome.jp/kegg/pathway.html). 
Bioinformatic analysis. To identify the candidate genes, we further analyzed the results using standard filters steps: (a) First, we excluded the variants that did not pass the quality filter. (b) Then, we excluded variants that were not protein-coding or splicing sites. Synonymous variants were also excluded. (c) We excluded variants in 1000 genomes or gnomAD or ExAC at frequency $\geq 0.001(7,8,10)$. (d) We screened the deleterious variants by $\geq 3$ in silico prediction tools for missense variants or frameshift variants. (e) We then presented the functional or bibliographical terms filter. (f) We screened the known CRC susceptibility genes including $A P C, M S H 2$, and PTEN (Supplemental Table 11). Individuals were selected if they carried the deleterious variants among these known genes. The other individuals did not carry the known CRC germline mutation genes for next step screen. (g) Variants were screened by manual reviews using the filtering standards: rare nonsynonymous and possibly damaging variants in DDR genes or participating in cell apoptosis, autophagy, cell cycle, cell growth, cell proliferation, angiogenesis, inflammatory response, cell differentiation, cell adhesion, and chromatin modification function. (h) We filtered the prioritize variants that fulfilled previous criteria - with interesting gene function and interactions - and were located in protein domains. (i) The filtered candidate genes were listed for further analysis. The screening flow chart is shown in Supplemental Figure 3.

Somatic mutation screening with WES. DNA extraction from formalin-fixed paraffin-embedded tissue was performed with the QIAamp DNA FFPE Tissue Kit (Qiagen) according to manufacturer's instructions. The methods of WES and annotations were performed as described above. KRAS, BRAF, and FBXW7 mutations and a total of 83 cancer-related genes were screened, including APC, CTNNB1, and PIK3CA (Supplemental Table 9).

Sanger sequencing of the candidate genes. The candidate pathogenic variants were verified by Sanger sequencing. One hundred healthy controls and 352 CRC/CRAs were also tested for the candidate variant using Sanger sequencing. The entire CHEK2 coding sequence was also performed by Sanger sequencing. Primer sequences are given in Supplemental Table 12.

IHC. Immunostains for Chk2 protein expression were performed on $4 \mu \mathrm{m}$ sections from colon tumor and normal mucosa from family $\mathrm{K}$ and $\mathrm{CRC}$ control or healthy control. After deparaffinization, citrate buffer was used to retrieve antigen. Endogenous peroxidase was inactivated with $3 \% \mathrm{H}_{2} \mathrm{O}_{2}$ dilution for 15 minutes. Tissues were blocked with 10\% goat serum for 1 hour at room temperature. The Chk2 primary antibody (ab109413, diluted at 1:100, Abcam) was incubated overnight at $4^{\circ} \mathrm{C}$. The goat anti-rabbit/mouse secondary antibody (Thermo Fisher Scientific, D-3004) was incubated for 1 hour at room temperature and subsequently revealed with DAB substrate (Dako) for 3 minutes. Slides were finally stained in hematoxylin. Immunostains for MSH2 (Thermo Fisher Scientific, diluted at 1:150, 33-7900), MSH6 (Abcam, diluted at 1:150, ab92471), PMS2 (Abcam, diluted at 1:100, ab110638), and MLH1 (Abcam, diluted at 1:100, ab92312) proteins expressions were performed as described above.

Functional characterization of genetic variants. The SW480 human CRC cell line was purchased from American Type Culture Collection (ATCC) and was cultured in 1640 medium supplemented with 10\% FBS (Thermo Fisher Scientific) and 1\% penicillin-streptomycin solution (MilliporeSigma). Cells were maintained in a humidified incubator adjusted with $5 \% \mathrm{CO}_{2}$ at $37^{\circ} \mathrm{C}$.

Establishment of CRISPR-KO cells. In order to generate CHEK2 ${ }^{\mathrm{KO}}$ mutant cells, the CRISPR-Cas9 system was used in accordance with manufacturer's protocol. The CRISPR designing tool (http://www.rgenome.net/cas-designer/) was used to design the single guide RNA (sgRNA). The sequences were used as list in Supplemental Table 12. The sgRNA was cloned into the pSpCas9(BB)-2A-GFP (PX458) vector and transiently transfected into the SW480 CRC cell line using FuGENE HD transfection reagent (Promega). Forty-eight hours later, transfected cells were plated onto 96-well plates for single cell cloning. After 2 weeks, genomic DNA was extracted from each clone and subjected to PCR amplification. The positive clones were screened by Sanger sequencing. The expression of Chk2 of the positive clones was verified by Western blot.

Protein extraction, Western blot, and antibodies. Whole-cell protein extracts were prepared with RIPA buffer supplemented with complete Protease Inhibitor Cocktail (Roche Life Sciences) and quantified using BCA protein assay kit (Thermo Fisher Scientific). Equal aliquot of protein lysate was run on a Tris protein gel electrophoresis and transferred onto a PVDF membrane (MilliporeSigma), according to manufacturer's protocols. Proteins were blocked in 5\% BSA for 1 hour and blotted with the indicated primary antibodies, which were diluted with $5 \% \mathrm{BSA}$ at $4^{\circ} \mathrm{C}$ overnight. Secondary antibodies were labeled and detected using ECL Kit (Pierce Biotech) by ChemiDoc Touch Imaging System (Bio-Rad) as described previously (47).

The antibodies were used as follows: anti-Chk2 (Abcam, diluted at 1:5000, ab109413); anti-phospho- $\gamma$ H2AX (phospho Ser139; Cell Signaling Technology [CST], diluted at 1:1000, 9718); anti-p53 (Abcam, 
diluted at 1:1000, ab32389); anti-p53 (phospho Ser20; Abcam, diluted at 1:1000, ab157454); and anti- $\beta$-actin (KangChen, diluted at 1:3000, KC-5A08).

Cell proliferation. The proliferation ability of cells was measured using the Cell Counting Kit-8 (CCK8) kit. Cells were inoculated into 96-well plates at a density of 2000 cells per well in sextuplicate. In total, $10 \mu \mathrm{L}$ of CCK8 aqueous reagent and $90 \mu \mathrm{L} 1640$ median was added to each well after 24, 48, 72, or 96 hours, respectively. After incubation at $37^{\circ} \mathrm{C}$ for 2 hours, the absorbance was read at $450 \mathrm{~nm}$ with an Epoch Microplate Spectrophotometer. All experiments were repeated 3 times.

Crystal violet assay. Cells were inoculated into 24-well plates at a density of $2 \times 10^{4}$ cells per well. After being treated with nocodazole (MedChenExpress, 31430-18-9) or Adriamycin (Selleck Chemicals, S1208) for 48 hours, cells were fixed with $4 \%$ formaldehyde for 20 minutes and dyed with $0.05 \%$ crystal violet for 20 minutes. All experiments were repeated 3 times. The ImageJ (NIH) software was used to calculate the normalized cell population in each well.

DNA damage. DNA damage was accessed in the presence of nocodazole with a concentration range from $0.8 \mu \mathrm{M}$ to $2 \mu \mathrm{M}$ or Adriamycin with a concentration range from $0.5 \mu \mathrm{M}$ to $6 \mu \mathrm{M}$ for a certain amount of time such as 24 hours or 48 hours. Cell lysates were collected and subjected to SDS-PAGE for Western blot analysis using primary antibodies.

Apoptosis and cell cycle assays. Cell apoptosis and cell cycle were measured after being treated with nocodazole or Adriamycin at the indicated time. Apoptotic cells were detected by labeling the samples with FITC-annexin $\mathrm{V}$ and propidium iodide (PI) (556547, BD Biosciences) in accordance with the manufacturer's protocol. For cell cycle analysis, cells were washed in phosphate-buffered saline and vortexed while adding $75 \%$ ethanol drop-wise to fix them. Cells were incubated at $4^{\circ} \mathrm{C}$ overnight. After that, cells were centrifuged at $400 \mathrm{~g}$ at $4^{\circ} \mathrm{C}$ for 5 minutes and resuspended in PBS containing $400 \mu \mathrm{L}$ PI (550825, BD Biosciences). Cells were incubated at $37^{\circ} \mathrm{C}$ in the dark for 20 minutes before flow cytometry analysis. Samples were analyzed on a FACS Calibur flow cytometer (BD Biosciences); the FlowJo vision 10 software and the Modfit LT software were used to define apoptotic cells or number of cells in each cell cycle.

Accession numbers. The accession numbers for CNVs reported in this paper are ClinVar: SCV001754535, SCV001754757, SCV001754759, SCV001754761, SCV001754762， SCV001754763， SCV001754805, SCV001754806, SCV001754807, SCV001754808, and SCV001754809.

Statistics. Data from at least 3 independent experiments performed are presented as the mean \pm SEM. Measurement of data between 2 groups was performed using nonparametric Mann-Whitney $U$ test. Statistical tests were 2 -tailed, and a $P$ value less than 0.05 was considered statistically significant. SPSS statistical software was used for analyses. Graphs and associated statistical analyses were performed using GraphPad Prism version 8 for Windows (GraphPad Software).

Study approval. This study was approved by the institutional ethics committee (KY2019-007) of Renji Hospital, School of Medicine, Shanghai Jiao Tong University, as well as by the Chinese National Review Committee for Genetics Studies. A written informed consent was signed by each participant.

\section{Author contributions}

PX contributed experimental design and conducted experiments, data analyses, and manuscript writing. DS contributed experimental design and data analyses, as well as manuscript writing. YG and YJ performed experiments. GZ, MZ, JC, ZW, and QL provided colon cancer specimens and clinical and pathological information. $\mathrm{JH}$ and HC discussed the data. PX, DS, and JYF conceived of the study and wrote the manuscript. YXC and JYF designed or/and supervised this project and revised the manuscript. The order of the co-first authors was based on the contributions to the work.

\section{Acknowledgments}

We thank all patients and individuals for their participation in our study. The authors thank Feng Zhang for useful advices. This project was supported in part by grants from State Key R\&D Program (no. 2020YFA0509200), the National Natural Science Foundation of China (nos. 81421001, 81530072, 81830081, 81902365, 81972203, 31970718, 81874047), Shanghai Municipal Health Commission, Collaborative Innovation Cluster Project (no. 2019CXJQ02), and Innovative research team of high-level local universities in Shanghai. 
1. Lichtenstein P, et al. Environmental and heritable factors in the causation of cancer--analyses of cohorts of twins from Sweden, Denmark, and Finland. N Engl J Med. 2000;343(2):78-85.

2. Czene K, et al. Environmental and heritable causes of cancer among 9.6 million individuals in the Swedish Family-Cancer Database. Int J Cancer. 2002;99(2):260-266.

3. Yurgelun MB, et al. Cancer susceptibility gene mutations in individuals with colorectal cancer. J Clin Oncol. 2017;35(10):1086-1095.

4. Jasperson KW, et al. Hereditary and familial colon cancer. Gastroenterology. 2010;138(6):2044-2058.

5. Manolio TA, et al. Finding the missing heritability of complex diseases. Nature. 2009;461(7265):747-753.

6. Valle L. Recent discoveries in the genetics of familial colorectal cancer and polyposis. Clin Gastroenterol Hepatol. 2017;15(6):809-819.

7. Palles C, et al. Germline mutations affecting the proofreading domains of POLE and POLD1 predispose to colorectal adenomas and carcinomas. Nat Genet. 2013;45(2):136-144.

8. Weren RD, et al. A germline homozygous mutation in the base-excision repair gene NTHL1 causes adenomatous polyposis and colorectal cancer. Nat Genet. 2015;47(6):668-671.

9. Adam R, et al. Exome sequencing identifies biallelic MSH3 germline mutations as a recessive subtype of colorectal adenomatous polyposis. Am J Hum Genet. 2016;99(2):337-351.

10. Nieminen TT, et al. Germline mutation of RPS20, encoding a ribosomal protein, causes predisposition to hereditary nonpolyposis colorectal carcinoma without DNA mismatch repair deficiency. Gastroenterology. 2014;147(3):595-598.

11. Olkinuora A, et al. Biallelic germline nonsense variant of MLH3 underlies polyposis predisposition. Genet Med. 2019;21(8):1868-1873.

12. Yan HHN, et al. RNF43 germline and somatic mutation in serrated neoplasia pathway and its association with BRAF mutation. Gut. 2017;66(9):1645-1656.

13. Bonjoch L, et al. Germline mutations in FAF1 are associated with hereditary colorectal cancer. Gastroenterology. 2020;159(1):227-240.

14. Golubicki M, et al. Germline biallelic Mcm8 variants are associated with early-onset Lynch-like syndrome. JCI Insight. 2020;5(18):e140698

15. Fearon ER. Molecular genetics of colorectal cancer. Annu Rev Pathol. 2011;6:479-507.

16. Carethers JM, Jung BH. Genetics and genetic biomarkers in sporadic colorectal cancer. Gastroenterology. 2015;149(5):1177-1190

17. Hirao A, et al. DNA damage-induced activation of p53 by the checkpoint kinase Chk2. Science. 2000;287(5459):1824-1827.

18. Shieh SY, et al. The human homologs of checkpoint kinases Chk1 and Cds1 (Chk2) phosphorylate p53 at multiple DNA damage-inducible sites. Genes Dev. 2000;14(3):289-300.

19. Chehab NH, et al. Chk2/hCds1 functions as a DNA damage checkpoint in G (1) by stabilizing p53. Genes Dev. 2000;14(3):278-288.

20. AlDubayan SH, et al. Inherited DNA-repair defects in colorectal cancer. Am J Hum Genet. 2018;102(3):401-414.

21. Mamrak NE, et al. Recent discoveries in the molecular pathogenesis of the inherited bone marrow failure syndrome Fanconi anemia. Blood Rev. 2017;31(3):93-99.

22. Niedzwiedz W, et al. The Fanconi anaemia gene FANCC promotes homologous recombination and error-prone DNA repair Mol Cell. 2004;15(4):607-620.

23. Rogers CD, et al. Genetics of the FANCA gene in familial pancreatic cancer. J Med Genet. 2004;41(12):e126.

24. Juko-Pecirep I, et al. Evaluation of Fanconi anaemia genes FANCA, FANCC and FANCL in cervical cancer susceptibility. Gynecol Oncol. 2011;122(2):377-381.

25. Erkko H, et al. A recurrent mutation in PALB2 in Finnish cancer families. Nature. 2007;446(7133):316-319.

26. Jones S, et al. Exomic sequencing identifies PALB2 as a pancreatic cancer susceptibility gene. Science. 2009;324(5924):217.

27. Girard E, et al. Familial breast cancer and DNA repair genes: insights into known and novel susceptibility genes from the GENESIS study, and implications for multigene panel testing. Int J Cancer. 2019;144(8):1962-1274.

28. Segui N, et al. Germline mutations in FAN1 cause hereditary colorectal cancer by impairing DNA repair. Gastroenterology. 2015;149(3):563-566.

29. Frank B, et al. Colorectal cancer and polymorphisms in DNA repair genes WRN, RMI1, and BLM. Carcinogenesis. 2010;31(3):442-445.

30. Broberg K, et al. Association between polymorphisms in RMI1, TOP3A, and BLM and risk of cancer, a case-control study. BMC Cancer. 2009;9:140.

31. Tischkowitz M, et al. Rare germline mutations in PALB2 and breast cancer risk: a population-based study. Hum Mutat. 2012;33(4):674-680.

32. Foo TK, et al. Compromised BRCA1-PALB2 interaction is associated with breast cancer risk. Oncogene. 2017;36(29):4161-4170

33. Xia B, et al. Fanconi anemia is associated with a defect in the BRCA2 partner PALB2. Nat Genet. 2007;39(2):159-161.

34. Pearlman R, et al. Prevalence and spectrum of germline cancer susceptibility gene mutations among patients with early-onset colorectal cancer. JAMA Oncol. 2017;3(4):464-471.

35. Bonache S, et al. Multigene panel testing beyond BRCA1/2 in breast/ovarian cancer Spanish families and clinical actionability of findings. J Cancer Res Clin Oncol. 2018;144(12):2495-2513.

36. Bartek J, et al. CHK2 kinase — a busy messenger. Nat Rev Mol Cell Biol. 2001;2(12):877-886.

37. Stolz A, et al. Tumor suppressor CHK2: regulator of DNA damage response and mediator of chromosomal stability. Clin Cancer Res. 2011;17(3):401-405.

38. Bell DW, et al. Heterozygous germ line hCHK2 mutations in Li-Fraumeni syndrome. Science. 1999;286(5449):2528-2531.

39. Kilpivaara O, et al. CHEK2 variant I157T may be associated with increased breast cancer risk. Int J Cancer. 2004;111(4):543-547.

40. Szymanska-Pasternak J, et al. CHEK2 variants predispose to benign, borderline and low-grade invasive ovarian tumors. Gynecol Oncol. 2006;102(3):429-431.

41. Pritchard CC, et al. Inherited DNA-repair gene mutations in men with metastatic prostate cancer. NEngl J Med. 2016;375(5):443-453.

42. Carlo MI, et al. Prevalence of germline mutations in cancer susceptibility genes in patients with advanced renal cell carcinoma. JAMA Oncol. 2018;4(9):1228-1235.

43. AlDubayan SH, et al. Association of inherited pathogenic variants in Checkpoint Kinase 2 (CHEK2) with susceptibility to testicular 
germ cell tumors. JAMA Oncol. 2019;5(4):514-522.

44. Kilpivaara O, et al. CHEK2 I157T associates with familial and sporadic colorectal cancer. J Med Genet. 2006;43(7):e34.

45. Bellido F, et al. Association between germline mutations in BRF1, a subunit of the RNA polymerase III transcription complex, and hereditary colorectal cancer. Gastroenterology. 2018;154(1):181-194.

46. Navon Elkan P, et al. Mutant adenosine deaminase 2 in a polyarteritis nodosa vasculopathy. N Engl J Med. 2014;370(10):921-931.

47. Yu T, et al. Fusobacterium nucleatum promotes chemoresistance to colorectal cancer by modulating autophagy. Cell. 2017;170(3):548-563. 